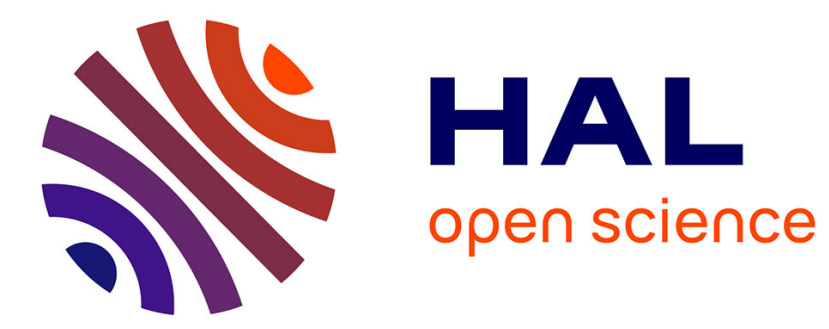

\title{
LA MORT DE MAX JACOB : RÉALITÉ ET REPRÉSENTATIONS
}

\author{
Patricia Sustrac
}

\section{To cite this version:}

Patricia Sustrac. LA MORT DE MAX JACOB : RÉALITÉ ET REPRÉSENTATIONS. Les Cahiers Max Jacob, 2009, 9, pp.103-118. hal-01806614

\section{HAL Id: hal-01806614 https://hal.science/hal-01806614}

Submitted on 9 Jun 2018

HAL is a multi-disciplinary open access archive for the deposit and dissemination of scientific research documents, whether they are published or not. The documents may come from teaching and research institutions in France or abroad, or from public or private research centers.
L'archive ouverte pluridisciplinaire HAL, est destinée au dépôt et à la diffusion de documents scientifiques de niveau recherche, publiés ou non, émanant des établissements d'enseignement et de recherche français ou étrangers, des laboratoires publics ou privés. 


\section{LA MORT DE MAX JACOB : RÉALITÉ ET REPRÉSENTATIONS}

Patricia SUSTRAC*

T a disparition de Max Jacob a privé la littérature française d'une de ses plus tances de sa mort, le 5 mars 1944 à Drancy, continuent d'apparaître comme le parachèvement d'une existence marquée par le martyre et pour beaucoup, par exemple pour le récent Prix Nobel, il y a « comme un symbole dans ce destin, qui est fait de cette double rencontre : la rencontre de Max Jacob avec son Ange, un jour de septembre 1909, dans "l'obscure petite chambre" qui bouleversa toute sa vie et le convertit au christianisme et, trente ans plus tard, la rencontre de l'humiliation raciale et de la mort nazie dans le camp de Drancy $»^{1}$. Une forte mobilisation rassembla les amis du poète pour tenter d'obtenir une mesure de clémence en sa faveur. Mais Max Jacob ne fut jamais libéré. Or, la conviction d'une libération doublée de l'idée qu'elle aurait été accordée le lendemain de sa mort est une certitude - erronée - chez ses biographes et persiste encore aujourd'hui, suscitant de vives polémiques quant à l'insuffisance des actions menées par Jean Cocteau, Sacha Guitry, et la défection de Pablo Picasso ${ }^{2}$. Nous proposons de montrer l'origine de cette erreur et la signification de ces débats dont les termes ne semblent pas encore aujourd'hui apaisés. Par ailleurs, nous essayerons de montrer que les récits de la mort du poète s'inscrivent dans un ensemble de textes hagiographiques, faisant

\footnotetext{
* Présidente de l'A.M.J., Patricia Sustrac travaille à la fois sur des questions biographiques et sur les correspondances du poète. Elle a publié Les Lettres de Max Jacob à Roger Toulouse, Troyes : Les Cahiers Bleus, 1992 (en collaboration avec Christine Van Rogger-Andreucci); Lettres à un jeune homme, 1938-1944, Paris : Bartillat, 2009 (lettres à Jean-Jacques Mezure).
} 
abstraction de la perspective historique, qui prolongent et fixent la réception des biographies écrites du vivant de l'artiste enrôlant faussement Max Jacob dans l'esprit de la Résistance.

\section{MAX JACOB N'A JAMAIS ÉTÉ LIBÉRÉ}

Dans les heures qui suivront l'arrestation, la mobilisation en faveur de la libération du poète s'est organisée ${ }^{3}$. Suite aux instructions de Max Jacob ${ }^{4}$ ou de leur propre initiative, des habitants de Saint-Benoît et ses jeunes amis de l'Orléanais préviennent André Salmon, Jean Cocteau et Conrad Moricand ${ }^{5}$. Il se dégage rapidement une double stratégie : d'une part, une action diplomatique sera menée auprès de l'ambassade d'Allemagne à laquelle Jean Cocteau et Sacha Guitry apporteront leur caution ; d'autre part, des actions auraient été menées par Sacha Guitry, Conrad Moricand et José-Maria Sert auprès du service des Affaires juives de la Gestapo.

Georges Prade pilotera l'action diplomatique. Membre de l'Action française avant la guerre, élu au Conseil municipal de Paris en 1929, vice-président du Conseil général de la Seine en 1934, Georges Prade est un homme d'affaires sans scrupules. Administrateur des Nouveaux Temps depuis 1941, pressenti, un temps, par les Allemands pour administrer Paris-Soir et Paris-Midi, il dirige également une société de publicité importante. Les nombreuses enquêtes de police menées à son sujet pendant son exil helvétique après la Libération relèveront que « depuis la veille de la guerre [il était] une personnalité politique et mondaine très parisienne, familier des grands restaurants et des stations touristiques en vogue. Il menait alors un train de vie important. Il avait de nombreuses relations parmi les fournisseurs de mode et les commerçants de luxe et dans les milieux artistiques et mondains $\nu^{6}$. De 1940 à 1944, ses fonctions politiques et commerciales le mènent à fréquenter autant les autorités allemandes au plus haut niveau que les milieux mafieux parisiens. Le 25 février 1944, il est sollicité par Conrad Moricand pour sauver Max Jacob, arrêté la veille. Quelques semaines auparavant, c'était Pierre Minet qui l'avait saisi pour Myrté-Léa, sœur cadette du poète, prisonnière à Drancy, dont Max Jacob espérait ardemment la libération?

Georges Prade chercha d'emblée à rassembler des personnalités dont le capital symbolique permettrait de souligner la notoriété littéraire de Max Jacob afin d'obtenir sa libération. Jean Cocteau rédigea : «Une page sur Max pour [le conseiller Hans Henning Von Bose] chef des prisons juives. $»^{8}$ Georges Prade suggérait : « d'agir rapidement pour éviter un nouvel éloignement qui risquerait d'être défi- 
nitif » et assurait son interlocuteur d' « une conversion sincère au catholicisme, un détachement complet des questions politiques et un âge déjà avancé justifi[ant] vraiment pareille mesure $»{ }^{9}$.

Le conseiller Von Bose ne fut jamais le « chef des prisons juives ». De 1940 à 1944, il occupa les fonctions de responsable du service juridique de l'ambassade d'Allemagne. Si Von Bose est un des interlocuteurs privilégiés de l'homme d'affaires, il n'a aucune compétence pour faire libérer Jacob. Au mieux est-il un relais. Aussi transmet-t-il ce dossier à Karl Otto Klingenfuss, ancien responsable des Affaires Juives des services spéciaux berlinois ${ }^{10}$. Conseiller de légation, il doit instruire, en amont, toutes les sollicitations qui ne devaient pas manquer d'être présentées à l'ambassadeur Otto Abetz. Lui a-t-il soumis la requête ? A-t-elle été transmise au service des Affaires Juives de la Gestapo ? Il n'a pas été retrouvé de navette de transmission de l'ambassade à la Gestapo, unique service instructeur in fine, seul à pouvoir ordonner la libération.

Conrad Moricand « [se foutra] à l'eau à [son] tour en allant voir le Hauptsturmführer Rodje [Roethke, chef du service des Affaires Juives de la Gestapo]..., un des plus grands rouages pour ces questions $»^{11}$. Il affirme s'être présenté « quatre fois » au siège des services et lui avoir écrit. Moricand attendait toujours son rendez-vous le 13 mars 1944, date à laquelle la nouvelle du décès est diffusée. Henri Jadoux ${ }^{12}$, fidèle assistant et biographe de Sacha Guitry, relate que le dramaturge et lui-même ont été entendus par les services de la Gestapo sans préciser le rang de l'autorité qui les reçut. Sacha Guitry, quant à lui, ne fait pas mention de cette rencontre, ni d'aucune autre démarche spécifique pour sauver Max Jacob ${ }^{13}$.

José Maria Sert, peintre officiel du régime franquiste, représentant l'Espagne auprès du Vatican, avait déjà obtenu des libérations, en particulier celle de Maurice Goudeket, le mari de Colette. Alerté par Misia, son épouse, Sert « sans penser une seconde à tous les ennuis qu'il pouvait s'attirer personnellement en intervenant en faveur d'un Juif sous la terreur allemande, mit immédiatement en branle toutes les influences qu'il était capable de faire jouer $»^{14}$. Selon elle, il finit par obtenir un ordre de libération qui arriva trop tard. Il n'a pas été retrouvé de trace de l'action de l'ambassade d'Espagne auprès du service des Affaires Juives de la Gestapo. Aucune trace n'existe dans les archives espagnoles ou dans celles des autorités allemandes qui répondaient dans ce genre d'affaires uniquement aux sollicitations émanant de personnalités allemandes de très haut rang ou d'ambassades étrangères ${ }^{15}$. 
Si nous ne possédons pas de trace écrite d'actions menées auprès du service des Affaires Juives de la Gestapo, cette inexistence n'implique pas une absence de démarches. On sait, par exemple, que Jean Paulhan fut prévenu par téléphone d'une visite de la police allemande. Il put fuir in extremis par les toits de la rue des Arènes. Mais, s'agissant d'une libération qui implique obligatoirement l'établissement de papiers d'identité et d'autorisations de sortie du camp, toute action, orale, secrète ou officielle aurait nécessairement conduit, lors d'une étape au moins de l'instruction, à l'émission par l'occupant d'un ou plusieurs documents administratifs permettant un élargissement définitif de Drancy. À de rarissimes occasions, des déportés ont pu être libérés. Les registres du camp, les autorisations de circulation, les courriers nombreux visés par plusieurs services et administrations diverses (y compris françaises) accompagnent minutieusement la libération de l'intéressé(e). Aucun document de ce type concernant Max Jacob n'existe dans les archives françaises ou allemandes, à aucune étape de la chaîne de l'instruction administrative usuelle pour un dossier de cette nature.

Comment, alors, expliquer la conviction tenace d'une libération du poète ? Plusieurs éléments peuvent être évoqués :

1/ Une conjonction de date fortuite : lorsque le conseiller Von Bose débute l'instruction de l'affaire Jacob, il transmet les documents en sa possession ${ }^{16}$. Cette transmission datée du lundi 6 mars a été considérée, à tort, comme l'avis de libération. La mort de Max Jacob étant survenue la veille, cette conjonction de date a par ailleurs induit que les Allemands avaient d'autant plus volontiers obtempéré qu'ils libéraient un cadavre. Cependant, le circuit de diffusion de l'acte de décès n'inclut aucune autorité allemande (déclaré le lundi 6 mars au commissariat de police de Pantin, le 7 à la mairie de Drancy, puis au Parquet, l'acte officiel d'état civil arrivera en mairie de Saint-Benoît le lundi 13 mars).

2/ La conviction du succès : qui ne fait aucun doute ${ }^{17}$, mais, " [1'affaire] est d'une délicatesse extrême à manœuvrer car il [ne] faut pas (...) indisposer les autorités allemandes dont tout dépend $»^{18}$. Les autorités délivrent cependant des informations. Par exemple, Georges Prade aurait obtenu, lors de son entretien le 25 février à l'ambassade, que Max Jacob, encore détenu à Orléans, « soit mis à un régime de faveur, c'est-à-dire dans une villa en dehors du camp, ce qui est appréciable tant pour le logement que pour la nourriture $»^{19}$. Existait-il près de la prison d'Orléans une « villa » confortable pour les juifs ? Le plan de la ville occupée et en ruines reste désespérément muet. Marguerite Toulouse, quant à elle, est « réconfortée » par le gros Willy, officier de la Gestapo d'Orléans : son ami «est là pour un contrôle ». Et le jeune Roger Toulouse qui s'est rendu à Paris pour solliciter 
l'intervention de son admirateur Albert Buesche, critique d'art au Pariser Zeitung, reçoit de sa part une réponse encourageante : « J'ai fait la démarche dont je vous avais parlé et on m'a dit que votre ami a bonne chance ${ }^{20}$. »

Quelle « bonne chance » Max Jacob avait-il d'être libéré ? Georges Prade qui, à cette époque, semblait être le « seul [qui pouvait] faire quelque chose et, en son temps, avait garanti sa tête $\aleph^{21}$, de son exil helvétique d'où il ne manquait pas de faire valoir son attitude héroïque pendant l'Occupation en rappelant les libérations de centaines de détenus dont « un bon contingent d'Israélites »" , ne cita jamais Max Jacob parmi eux ${ }^{23}$.

Les réponses apportées par les autorités allemandes abusèrent les consciences. Aucun de ceux qui œuvrèrent dans cette chaîne d'espérance ne pouvait concevoir que pour un Klingenfuss, ou pour un Alois Brunner, commandant impitoyable de Drancy, le poète, que tous aimaient affectueusement, était enseveli au cœur de l'idéologie raciale antisémite nazie ; que dès lors, il ne « valait » rien, qu'il n'était " rien », pas même un homme, pas même un chien, mais un Untermensch. Max Jacob est mort victime du génocide nazi, au terme d'un processus mis en œuvre en plusieurs étapes avec la complicité du régime de Vichy ${ }^{24}$ :

- La définition, l'identification et le repérage : Max Jacob « répondait » à la définition du Juif selon le Statut d'octobre 1940 ; il se déclara à la souspréfecture de Montargis, fut soumis au port de l'étoile jaune et privé de ses libertés individuelles.

- La spoliation économique : Max Jacob fut privé de ses droits d'auteur et était interdit de publication par décision des Listes $\mathrm{Otto}^{25}$. En tant que juif, ses rations étaient inférieures à celles des non-juifs.

- La déportation, la mort : Max Jacob fut déporté à Drancy, étape vers Auschwitz-Birkenau où il aurait dû être acheminé par le convoi no $69 \mathrm{du}$ 7 mars 1944 pour y être gazé.

La requête de Jean Cocteau nous semble devoir être envisagée comme le début d'une procédure de recevabilité d'une mesure de clémence ou de libération hypothétique de Max Jacob, mais en aucun cas comme une libération ${ }^{26}$. Déposée au sein d'un service qui n'avait aucune compétence d'instruction, transmise à un responsable impliqué dans l'extermination des Juifs au moment où le rythme des persécutions s'accroît pour que chaque convoi atteigne un quota de 1000 à 1200 déportés, la requête ne pouvait connaître d'autre fin que d'être classée définitivement le 8 avril 1944 au sein des services de l'ambassade dont elle n'est jamais sortie. 
C'est dans le cadre de l'historiographie de la Shoah que s'explique la mort du poète. Max Jacob est la victime d'un crime inouï, organisé et méthodiquement planifié lors de la conférence de Wannsee en janvier 1942 où le sort des Juifs d'Europe fut définitivement scellé par les dirigeants du Reich. S'abstraire de cette historiographie retire la responsabilité de ce crime à leurs auteurs, laisse place à des polémiques au cours desquelles les condamnations recoupent les poncifs des 《 bons » et des « mauvais » Françaiis ${ }^{27}$ pendant l'Occupation, et in fine soit assigne à la mort du poète un caractère immanent à l'œuvre (« l'aspiration au martyre » qui le mènera naturellement de la « sinistre mort allemande » du Cornet à dés à la prémonition de sa déportation), soit la définit comme la « rançon » d'une biographie mouvementée ${ }^{28}$. Nous reviendrons sur les traductions littéraires de ces conceptions qui présentent souvent comme signe distinctif de proposer un portrait hagiographique puis mythique du poète. Nous proposons, tout d'abord, d'envisager les sources de ces polémiques qui nous semblent issues de questions politiques à propos desquelles, en 1955, André Salmon déjà s'interrogeait : "'Les scoliastes futurs" devront-ils débattre d'un Mythe de Max Jacob ? Ce n'est pas impensable. [...]. [Son] martyre a favorisé la fabrication d'un Jacob de gauche. En choisissant les textes on pourrait obtenir un suffisant Max de droite. ${ }^{29}$

\section{LA MORT DE MAX JACOB : UNE AFFAIRE DE GAUCHE?}

Le 10 octobre 1944, lors d'un dîner, Christian Bérard portait ostensiblement un pantalon en velours de Max Jacob offert par Pierre Colle. Cocteau avait émis des réserves ; Pierre Colle les avait repoussées : «C'est un pantalon comme un autre. Max détestait le fétichisme ». Picasso, comprenant que Bérard portait le pantalon de Jacob, blêmit, s'insurgea et quitta la réception ${ }^{30}$. Ce qui aurait pu rester sur le plan de la morale prit cependant un tour politique. Quelques jours avant ce dîner, le 5 octobre, Picasso venait d'adhérer au Parti communiste français. Cet événement avait eu un grand retentissement : «Événement du mois », notait ironiquement Jean Cocteau dans son Journal : «L'Humanité lui consacr[e] toute la première page. La guerre pass[e] ensuite ». Cocteau poursuivait : «Picasso l'intouchable, extérieur à tout système, solitaire et glorieux, se trouve soudain appuyé par un ordre extérieur, abandonné par ce qui s'oppose à cet ordre, jugé sous l'angle politique. $"{ }^{31}$ Cet angle politique fut, selon Cocteau, le « prétexte à une mise au point communiste »: « Picasso possède une adresse profonde. Il choisit toujours entre mille la circonstance précise, capable d'aider sa haute propagande. » Le 20 octobre paraissait dans Combat un article indigné de Michel Leiris à propos de l'incident intitulé CEUXqui 
ne comprendront jamais. Cocteau pressentait-il les conséquences de l'article de Leiris dans le contexte politique du moment où chacun pensait déjà à répondre de ses responsabilités pendant 1'Occupation ? Il considéra d'emblée ce texte comme une incitation à la violence. Michel Leiris, selon lui, profitait de l'affaire pour raconter « l'histoire du pantalon de telle sorte qu'en province, où les esprits sont en feu, on nous lapiderait aveuglément en cinq minutes ». In fine, Cocteau assimilait ces méthodes aux « méthodes de police qui nous écœuraient dans la presse française d'Occupation ». Cocteau devait avoir perçu comment la mort de Max Jacob pouvait devenir un enjeu de distinction dans la frontière de la collaboration, aussi voulait-il le minimiser et le circonscrire : « Nous aimons un Max vivant et nous sommes poursuivis par ceux qui aiment un Max mort et qui organisent sa légende. » Il s'attachera à brocarder ceux qui « envisage[nt] une histoire de pantalon sous l'angle du drame » en lui donnant un tour politique qu'il condamne : "L'aventure Picasso, c'est la gauche à gauche. Grâce aux surréalistes. Grâce à Eluard et à Louis Aragon, voici de l'ordre, et tout ordre supérieur amène le scandale. " Picasso allait ultérieurement devenir la cible privilégiée des critiques concernant la mort de Max Jacob, en particulier celle de Pierre Andreu, journaliste et homme de lettres fortement ancré à droite, l'accusant d'avoir abandonné le poète ${ }^{32}$.

Pour Jean Cocteau, c'est dans un registre religieux qu'il allait donner à la mort de Max Jacob sa signification. Cette mort deviendra, le 15 mars 1944, date à laquelle il apprend le décès de son ami, une révélation surnaturelle rédemptrice qui le délivrera de son aveuglement des années d'Occupation. Énumérant pour luimême les termes de sa défense, afin de répondre aux accusations de son article en faveur d'Arno Breker, il notait :

J'ai fait cet article dans Comoedia par reconnaissance et parce que j'ai trouvé élégant de louer un ennemi comme un ami. Je m'en excuse. Paul Eluard m'a prouvé que j'avais tort et que cette élégance d'âme était inélégante par rapport à Kafka et autres écrivains que j'admire et que l'Allemagne poursuivait de sa haine. J'ai compris ma faute le jour de la mort de Max Jacob. ${ }^{33}$

La mort de Max Jacob servira également de « rappel à l'ordre ». Jean Paulhan redonnera quelques clés de lecture à un Jouhandeau décidément bien troublé par l'époque, en lui rappelant sans ménagements :

De ton courage, personne (ni surtout moi) ne doute. Mais, en ce moment, je t'en prie, n'en parle pas. Ouvre les yeux. Tu n'es pas exposé. Ce n'est pas toi qui es exposé. Ce n'est pas toi qui viens de mourir en prison, c'est Max Jacob. ${ }^{34}$ 


\section{MAX JACOB ET LA FIGURE DE LA SAINTETÉ}

La mort de Max Jacob a plongé ses amis dans un profond désarroi. « Ce n’est pas tant un mort que nous pleurons, il ne peut guère être qu'auprès de ce Dieu qu'il a tant aimé, mais nous sommes tristes parce que sa mort nous laisse seuls, dépourvus, désemparés, abandonnés, car nous n'aurons plus pour réchauffer notre foi l'ardent rayonnement de la sienne. $\rangle^{35} \mathrm{Au}$ cours de la conférence-hommage du 21 mars 1944 à Saint-Benoît, le docteur Durand témoigna avec émotion d'un « Max Jacob tiss[ant] sa vie d'apôtre ». Messager et prophète, il était «l'homme qui m'a fait penser à Dieu, l'homme qui m'a fait poser sérieusement le dilemme : Dieu estil présent ou non sur l'autel ? ». Ces termes semblent suggérer, au moins dans ce cercle amical, que le poète était considéré comme un saint : " La prière de Max Jacob soutenait au-dessus de nos têtes un pan de ciel trop lourd, il n'était voûté que de prier pour tous (...). Côté prière, sa présence continue, plus sensible peut-être de ne plus être marquée par la misérable apparence. Max Jacob prie encore pour tous, je le sens intensément. $\aleph^{36}$ Max Jacob témoignait, de son vivant, de la présence divine ; disparu, il intercéderait désormais pour les vivants égarés.

Considéré comme un saint, le récit de sa vie s'inscrira rapidement dans le genre littéraire de la légende. Racontant la Passion du martyr, la légende fera abstraction du contexte historique. Elle présentera, à partir du récit des éléments de sa vie, de son œuvre ce qui doit susciter l'admiration. Dans son article Pour une poétique de la légende : "la vie de Monsieur Pascal », Philippe Sellier démontre l'expansion du genre littéraire de la légende grâce au christianisme qui, le premier, a affirmé le caractère unique et irremplaçable de chaque personne humaine : «On aime scruter la vie de ceux qui ont suivi le chemin du Christ : les martyrs d'abord, les moines, les religieuses puis les laïcs. Tout naturellement, et surtout quand il s'agit de martyrs, on privilégie les «derniers jours », couronnement et vérité d'une existence », et l'auteur ajoute : "Les défaillances seront omises, puisqu'on veut dessiner la courbe d'ensemble d'une existence : refusant l'éparpillement factice des notations, la légende vise à faire découvrir la surréalité de la vie. $\aleph^{37}$ Les textes qui permettront de « recevoir les toujours trop mirifiques enluminures de la légende $»^{38}$ concerneront surtout les récits de la mort du poète. Ils imprimeront durablement les biographes jacobiens dans le traitement d'une double figure de Max Jacob, celle de «l'âme d'un saint et d'un martyr »" 


\section{LES RÉCITS DE LA MORT DE MAX JACOB}

Yanette Delétang-Tardif, la première, a publié le récit le plus étoffé de la mort de Max Jacob à Drancy ${ }^{40}$. Elle put rencontrer, dans les tout premiers mois qui suivirent la libération du camp le 17 août, le médecin-chef Raymond Weille. L'article donne les lignes directrices dont s'inspireront les chroniqueurs ultérieurs. La libération de Max Jacob « signée et annoncée » d'autant que « les Allemands libéraient un cadavre » apparaît pour la première fois dans cette publication. Cette erreur a perduré jusqu'à nos jours :

Dites-le bien : à l'infirmerie, il fut admirablement soigné par nous tous, ses derniers compagnons de détention, et entouré par nous, de tous les soins humainement possibles. Il voulait mourir. C'était son heure. Il disait : "Je suis avec Dieu ». Il ne parlait presque pas. Il ne demandait rien. Il ne souffrait pas. Il était si loin de nous qu'il n'avait rien à dire. Il priait ».

Il ne m'exprima à moi, en particulier, qu'un seul désir : il voulait mourir catholiquement. Avec quel tact, quelle discrétion il formula cette demande, pour ne pas nous froisser, nous Juifs. Il murmurait : "Vous comprenez, j'ai donné ma vie à cette Passion ». Nous lui promîmes tout. Nous arrivâmes à tenir notre promesse. Cela ne dura guère plus de vingt-quatre heures. Littéralement il s'éteignait, avec une soumission, une modestie extraordinaire. Il n'eut pas une révolte, pas un reproche, pas d'agonie. Il avait déjà dépassé toute lutte. Il paraissait heureux. Je crois, oui, je crois qu'il était heureux. [...] À quelle heure il mourut? Le matin, vraiment comme les lampes. C'était beaucoup plus que de la résignation, c'était une adhésion absolue à un départ et avec une simplicité, une grandeur inouïe...

J'écoute encore la voix. Cet homme qui est un médecin c'est-à-dire celui qui voit souvent mourir a été si frappé du signe de cette mort, qu'il me répète et répète toujours avec une sorte de surprise absolument fervente : Ce fut incroyable, il ne voulait plus que mourir!

Le ton de cet article donne à la mort du poète les accents d'une passion dont le chemin de croix restera une thématique fréquente dans les récits ultérieurs du calvaire jacobien ${ }^{41}$. Jacob y sera présenté habité d'une abnégation devant l'épreuve fatale : " Il regarde ses bourreaux sans haine », enchérira l'auteur anonyme d'un article paru le 5 septembre 1944 dans Ce Soir. Ces interprétations nous paraissent témoigner de la création d'une figure de sainteté, qui conduiront, par exemple, Pierre Lagarde, en 1944, à livrer un témoignage poignant sous le titre de Max Jacob, mystique et martyr ${ }^{42}$ dans lequel le récit de l'arrestation ou de la mort de Jacob se calquent sur les récits christiques : 
112 La mort de Max Jacob : réalité et représentations

Comment ne pas imaginer la scène? La petite maison tranquille de Saint-Benoîtsur-Loire, la place bordée de platanes, Max Jacob occupé à peindre ou à écrire ou à prier - et depuis longtemps, pour lui, peindre ou écrire, c'était prier - la voiture grise s'arrête, les appels, les ordres brutaux, le soudain désarroi du poète, le cour qui se met à battre si vite qu'il semble ne plus battre, et, presque aussitôt, la religieuse acceptation, la soumission chrétienne :

C'est moi.

La réponse même de Jésus au jardin des Oliviers. ${ }^{43}$

Et René Guy Cadou, en 1948, d'évoquer l'ascension céleste :

À l'infirmerie de Drancy, le 5 mars 1944
et tous ceux qui sont morts vous le diront
on a vu une étoffe bleue longtemps
flotter au-dessus de la salle. ${ }^{44}$

Cette figure de la sainteté conduira les auteurs à livrer des textes hagiographiques qui ne sont pas uniquement inspirés par la mort de Max Jacob. Ils puisent aussi dans un corpus biographique préexistant. Ainsi, on peut relever le parallélisme du titre de l'ouvrage de Pierre Lagarde en 1944 et celui d'André Salmon en 1927 : Max Jacob, Poète, Peintre, Mystique et Homme de qualitét5. Par ailleurs, comme il n'était pas rare que le poète ajoute des éléments au récit de son existence mouvementée (le jeu sur sa date de naissance, son enfance inspirée par la terre sainte de Bretagne, ses vies fabuleuses d'aventurier...), il tissera parfois des trames romanesques ${ }^{46}$ quelquefois reprises par des biographes complaisants comme ce fut le cas d'Hubert Fabureau :

Or, en ces temps-là, un peu de semence d'Abraham était tombé au giron de l'Armorique [...] où vivait, affranchie des rites ancestraux, mais rebelle encore à l'enseignement des apôtres, la postérité de Jacob, qui pour confondre le faux prophète s'enrichit sans plus tarder d'un nouveau rejeton. C'était le 11 juillet 1876. La joie fut grande en Israël. Cependant le Dieu des Chrétiens, dans son infinie miséricorde, veillait sur l'âme frêle du garçon vagissant et par des voies mystérieuses d'ahan la guidait au port.

Il y eut des soirs, il y eut des matins.

«La légende dorée du bienheureux Max Jacob », titre du premier chapitre de cette épopée biblique, ne pouvait qu'être placée sous l'exergue d'André Billy : « Saint Max Jacob! Et pourquoi pas ? » ${ }^{47}$. 


\section{MAX JACOB, POÈTE DE LA RÉSISTANCE}

Les obsèques solennelles de Max Jacob à Saint-Benoît-sur-Loire eurent lieu le 5 mars 1949. Jean Cassou, ami fidèle du poète, écrivain et résistant, rédigea l'oraison funèbre :

Comme Péguy faisait jadis présentation à N.-D. de Chartres de cette terre de France pour laquelle il allait donner sa vie charnelle, nous faisons aujourd'hui offrande à l'un des plus glorieux sanctuaires de notre pays des restes charnels d'un autre poète, lui aussi saint et martyr. Max Jacob reposera désormais à l'ombre de cette vénérable église et continuera d'y prier pour ses pauvres frères humains. Pour nous, en cet anniversaire du jour où son âme s'envola vers l'éternité, nous bornons notre ferveur à évoquer son existence terrestre, l'être prodigieux qu'il fut, l'ami délectable, l'esprit unique, paré de tous les dons, de toutes les fantaisies et de tous les charmes. Nous qui avons connu, qui avons aimé Max Jacob, nous savons que pareille combinaison de hasards naturels et surnaturels ne se produira plus de longtemps, et qu'il y eut là pour la joie de ses amis et pour la poésie française une rencontre miraculeuse de perfections et de merveilles. Ce mélange d'invention et de rigueur, de pitié et d'ironie, cette lucidité d'un impitoyable artiste, cette cocasserie d'un personnage shakespearien, cette dévotion d'un facétieux jongleur, tant de contradictions et tant de multiplicités, tant d'humble misère et tant d'étincelant génie, tout cela est sorti du Cornet à dés, par un même coup de veine et qui a fait notre fortune spirituelle. La bête, elle aussi, avait bien calculé son coup ; elle avait choisi sa victime en s'abattant sur ce chétif rossignol de chez nous. Le nom de Max Jacob restera inscrit en tête de la protestation implacable de l'esprit français, de l'esprit humain contre la brute.

En 1949, il est, semble-t-il, difficile, même pour un résistant de désigner autrement que par métaphores ce que venait de nommer le procès de Nuremberg en 1946 en désignant les crimes contre l'humanité perpétrés, par exemple, contre le peuple juif. En 1961, la mention « mort pour la France » fut attribuée, à titre civil, à Max Jacob, à la demande de Roger Secrétain, député-maire d'Orléans, ami du poète et membre du bureau de l'association de ses amis. Est-ce cette mention qui introduisit la confusion et permit de croire que Max Jacob en devint titulaire à titre militaire ? En 1996, Neal Oxenhandler dans son ouvrage Looking for Heroes in postwar France associera Albert Camus, Max Jacob et Simone Weil ${ }^{48}$ dans la même démarche d'insoumission enrôlant le poète dans l'esprit de la Résistance. De même, Olga Rosenbaum ${ }^{49}$ associera Max Jacob à la figure de résistants comme Jean Cassou, Jean Cayrol, Madeleine Riffaud, Pierre-Henry Simon, Philippe Soupault 


\section{La mort de Max Jacob : réalité et représentations}

qui tous choisirent des modes d'engagements allant de la publication clandestine à l'engagement armé. Mais Max Jacob ne participa à aucune de ces modalités. Sans doute, ces arrangements avec l'histoire tiennent-ils à une fausse interprétation de la question du martyre chez Max Jacob. Il est tout à fait exact que Jacob a aspiré au martyre, toutefois ce serait un contresens de considérer cette aspiration comme un acte politique. Le martyre s'articule chez le poète à la notion de la souffrance, don divin. Par son intervention l'homme se retrouve lui-même ; elle est une extraction violente des scories qui nous sont étrangères et nous dispersent de l'essentiel. Cette souffrance est une situation existentielle et non pas une morale de l'agir. Jacob, être souffrant, poursuivait dans l'espoir de la Rédemption, la quête absolue de lui-même. La guerre lui apporta une épreuve redoutable car elle le confrontera à la question cruciale de l'être et, paradoxalement, pour ce juif converti depuis 1915, de son êtrejuif : « J'suis l'bouquet/J'suis le bouquet/J'suis le bouc émissaire... qui est responsable de toute cette pourriture littéraire : c'est le juif destructeur ? $\|^{50}$. Homme de l'introspection, Max Jacob anticipa lucidement la question sartrienne : « Le Juif est un homme que les autres hommes tiennent pour Juif ; voilà la vérité simple d'où il faut partir [...] c'est l'antisémite qui fait le Juif. »

$\mathrm{Au}$ terme de nos recherches, une réponse nous échappe. Pourquoi le poète ne figure-t-il pas sur la liste des intellectuels sauvés par Varian Fry ? Mandaté en 1940 par l'Emergency Rescue Committee, muni d'une liste de deux cents écrivains et artistes parmi lesquels : Chagall, Ernst, Breton, Masson, Arendt, Bellmer, Duchamp, Peret, Ophüls... Fry organisera leur séjour à la villa Air-Bel à Marseille et les fera rejoindre les États-Unis ou le Portugal. L'écrivain allemand Thomas Mann et le théologien français Jacques Maritain, alors en exil en Amérique, se joignirent à d'autres figures du monde universitaire et intellectuel pour établir la liste de ceux qui se trouvaient pris «dans le plus gigantesque piège humain de l'Histoire ${ }^{51}$. Malgré nos demandes, il n'a pas encore été possible d'accéder aux archives américaines et de consulter les étapes préparatoires à la liste définitive. Par ailleurs, il n'a pas été possible non plus de savoir si les intellectuels français qui ne souhaitaient pas émigrer comme Gide, Malraux ou Matisse amendèrent ou non la liste définitive. Existait-il un « quota»? Quelles furent les pressions pour les inscriptions de complaisance ? Quels furent les critères d'éligibilité choisis ? Quelle influence reconnaissait-on au département d'État à Washington à tel ou tel intellectuel exilé pour lui autoriser « ses » protégés ? Ces questions restent, pour l'instant, en suspens. 


\section{CONCLUSION}

La mort de Max Jacob a privé ses amis et ses admirateurs d'un être précieux et l'histoire de l'art du $\mathrm{XX}^{\mathrm{e}}$ siècle d'une de ses plus grandes figures, en aucun cas elle ne le transforme en héros ou en résistant. Jacob est mort car il était né juif, issu d'une famille juive à l'ascendance de plus de trois générations, à une époque où ceci était non un fait de l'histoire individuelle dont le poète choisit de se détacher mais une faute contre laquelle une politique systématique d'extermination s'était mise en œuvre. À l'instar de tous les Juifs d'Europe, Max Jacob est une victime. Replacer sa mort dans le contexte historique permettra de désengager la figure de sainteté d'hagiographies hâtives. L'œuvre de Max Jacob en ressortira plus puissante que jamais car les poètes n'ont pas besoin de compassion.

\section{NOTES}

${ }^{1}$ LE CLÉZIO J. M. G., « Préface », dans JACOB Max, Derniers Poèmes en vers et en prose, Paris : Gallimard (coll. Poésie), 2003, p. 12.

${ }^{2}$ LACHENAL Lydie, « La mort de Max », La Nouvelle Revue Française, n 566, juin 2003, p. 91108 ; ANDREU Pierre, Max Jacob, Namur : Wesmael-Charlier (coll. Les conversions célèbres), 1962 ; Vie et Mort de Max Jacob, Paris : La Table Ronde, 1982 ; Monsieur Max, fiction de Gabriel Aghion, scénario de Dan Franck, Paris : ARTE/France, 2007.

${ }^{3}$ Voir le calendrier en dernière section de cet article et SUSTRAC Patricia, « La mort de Max Jacob, janvier-mars 1944, un calendrier fatidique », site http//www.max-jacob.com : Les Amis de Max Jacob, 2007, onglet arrestation.

${ }^{4}$ Un document autographe circa 1941 cite des protections : sa famille, Paul Petit, Picasso et Mme Wormser (archives Les Amis de Max Jacob).

${ }^{5}$ Conrad Moricand, personnage équivoque, fut probablement, à des périodes de sa carrière, un des protecteurs de Jacob. Quand Gaston est incarcéré en décembre 1941, le poète craint cependant de l'importuner (JACOB Max, Lettres à Théophile Briant et Conrad Moricand 1920-1941, correspondance annotée et présentée par Stanley J. Collier, Oxford : Blackwell, 1966, p. 137). Le 26 août 1942 quand il craint une arrestation, le poète évoque auprès de Moricand « une intervention puissante [qui] empêche cette arrestation » (ibid. p. 139). Mais Jacob est désolé quand il perdra sa place de journaliste : «La place qu'il avait m'a sauvé la vie ! il n'y est plus et je n'ai plus de protecteur ! » (à Robert Szigeti, 17 septembre 1943, ms inédit, Médiathèque d'Orléans) ; in fine, en 1944, Jacob constate qu'il ne peut rien (ibid. p. 140 et p. 145).

${ }^{6}$ Exposé du commissaire du gouvernement le 9 juin 1947 (dossier G. Prade, CARAN, Paris, 334 AP, 26).

${ }^{7}$ « Merci ! Dis à Georges Prade ma gratitude... On a des amis inconnus, ça fait plaisir. Qu'il m'envoie ses livres ! Je n'ose lui écrire pour ne pas l'importuner et abuser [...] j'ai été très touché que Georges Prade ait une gouache de moi » (JACOB Max, Lettres à Pierre Minet, correspondance annotée et présentée par Anne Kimball, Paris : Calligrammes, 1988, p. 118). 


\section{La mort de Max Jacob : réalité et représentations}

${ }^{8}$ COCTEAU Jean, Journal 1942-1945, texte établi présenté et annoté par Jean Touzot, Paris : Gallimard, 1989, p. 481.

${ }^{9}$ PRADE Georges, « Note à M. le Conseiller Von Bose du 29 février 1944», dans LACHGAR Lina, Arrestation et mort de Max Jacob, Paris : La Différence (coll. Littérature), 2004, p. 69. Pour les ressources documentaires concernant le décès du poète, voir également JACOB Max, L'Amitié, lettres à Charles Goldblatt, correspondance établie et présentée par André Roumieux, Bègles : Le Castor Astral, 1994.

${ }^{10} \mathrm{~K}$. Otto Klingenfuss a signé des directives de déportation des juifs de Serbie en 1942, NATANSON Dominique, «Que sont-ils devenus » dans Le procès de Nuremberg : les accusés, le verdict et les liens avec la Shoah, site Mémoire juive et éducation, 2008, notice 597.

${ }^{11}$ JACOB Max, Lettres à Théophile Briant et Conrad Moricand 1920-1941, op. cit., p. 151. Moricand évoque aussi auprès du docteur Szigeti « une monnaie d'échange ». Toute armée est évidemment corruptible mais il n'existe aucune trace d'une collecte de fonds, d'or ou d'œuvres d'art auprès des amis de Jacob qui aurait pu servir de monnaie d'échange contre sa libération (MORICAND Conrad, «Une lettre de Conrad Moricand transmise par le Docteur Szigeti », dans Cahiers Max Jacob, St-Étienne : Maison Rhône-Alpes des Sciences de l'Homme/Centre de recherche Max Jacob, n¹0, 1988, p. 61-66). ${ }^{12}$ JADOUX Henry, Sacha Guitry, Paris : Perrin, 1982, p. 115.

${ }^{13}$ GUITRY Sacha, Quatre ans d'Occupations, dans Cinquante ans d'Occupations, Paris : Presses de la Cité (coll. Omnibus), 1992, p. 707-899. À partir de 1942 « an terrible pour les Juifs », c'est « quotidiennement que [leurs] doléances [lui] parviennent » (ibid. p. 849). Si les démarches pour libérer Tristan Bernard sont très précisément décrites (ibid., p. 867-880), rien n'est noté en faveur de Max Jacob. ${ }^{14}$ GOLD Arthur et FIZDALE Robert, La vie de Misia Sert, Paris : Gallimard (coll. Folio), 1984, p. 342.

${ }^{15}$ Les recherches concernant l'instruction administrative de la mort du poète ont été menées auprès de différents services d'archives situés en France : CARAN (archives nationales), archives départementales du Loiret, du Finistère et du Val-de-Marne, Centre de Documentation Juive Contemporaine ; en Suisse : archives fédérales suisses à Berne (en particulier le dossier du département fédéral de justice et de police cote E4320(B)1991/243) ; en Allemagne : Auwärtiges Amt, Politisches Archiv à Berlin (archives R 58, direction de la sécurité du Reich ; R 70 : dossiers de la police allemande dans les pays conquis: France, NS 19 : équipe de direction de la SS, fond « National-socialisme des Archives du ministère de la sécurité de la RDA »). Nous remercions M. Gerhard Keiper de l'Auwärtiges Amt, Politisches Archiv de Berlin pour son aide précieuse.

${ }^{16}$ « Herrn Gesandtschaftsrat Klingenfuss Zuständigkeitshalber vorgelegt. Max Jacob, der mir als Dichter bekannt ist, ist $\mathrm{m}$ [eines] $\mathrm{W}$ [issens] 1/2 Jude,1/2 Bretone. 6. 3. B ». « Pour suite à donner : affaire confiée à Monsieur le conseiller consulaire Klingenfuss, Max Jacob, poète à ma connaissance, est moitié juif et moitié breton». 6 mars Von Bose ». Nous remercions Mme Yasmin Hoffman pour sa traduction.

${ }^{17}$ « Prade me téléphone que nous avons toutes les chances d'aboutir », COCTEAU Jean, Journal 1942-1945, op. cit., p. 484.

${ }^{18}$ JACOB Max, Lettres à Théophile Briant et Conrad Moricand 1920-1941, op. cit., p. 151.

${ }^{19}$ Ibid. p. 150.

${ }^{20}$ JACOB Max, Lettres à Roger Toulouse, 1937-1944, correspondance réunie et annotée par Patricia Sustrac et Christine Van Roger-Andreucci, Troyes : Les Cahiers Bleus, 1992, p. 101.

${ }^{21}$ MORICAND Conrad, « Une lettre de Conrad Moricand transmise par le Docteur Szigeti », op. cit., p. 63.

${ }^{22}$ Procès-verbal complémentaire de l'audition établi par l'inspecteur Pache le 12 mai 1950 dans VAN DONGEN Luc, Un Purgatoire très discret, la transition «helvétique » d'anciens nazis, fascistes et collaborateurs après 1945, Paris : Perrin, 2008, p. 458. 
${ }^{23}$ « J'ai sa dernière lettre. Il me l'avait écrite du train qui l'emmenait à Drancy et confiée à un garde-mobile. Un collaborateur craintif et qui voulait se pré-blanchir l'a sauvé mais trop tard, il était mort » COCTEAU Jean, Le passé défini, 1951-1952, journal, Paris : Gallimard, 1983, T. I, p. 62 .

${ }^{24}$ Se reporter aux travaux de HILBERG Raul, La destruction des Juifs d'Europe, Paris : Gallimard (coll. Folio), 2006, 3 volumes ; BROWNING Christopher (avec les contributions de Jürgen Matthäus), Les origines de la solution finale, l'évolution de la politique antijuive des nazis, septembre 1939-mars 1942, Paris : Les Belles Lettres, 2007 ; BENSOUSSAN Georges, Europe, une passion génocidaire, essai d'histoire culturelle, Paris : Mille et une Nuits, 2006.

${ }^{25}$ Les Listes Otto interdisent les ouvrages « qui par leur esprit mensonger et tendancieux ont systématiquement empoisonné l'opinion française, en particulier les publications de réfugiés politiques ou d'écrivains juifs qui, trahissant l'hospitalité que la France leur avait accordée, ont sans scrupules poussé à une guerre dont ils espéraient tirer profit pour leurs buts égoïstes » (Préambule, Archives Nationales AJ40/1005).

${ }^{26}$ Brigade de la gendarmerie de Gien, procès-verbal des états des arrestations opérées par les autorités allemandes entre le 5 et le 15 mars 1944 : « Max Jacob (juif) arrêté le 24 février : motif de l'arrestation : ignoré. État des libérations pendant la même période : « néant » (JACOB Max, Les Amitiés et les Amours, op. cit, T. III, p. 102).

${ }^{27}$ « Pendant la guerre, il y avait ceux qui étaient planqués, qui ne pouvaient plus écrire et qui ne mangeaient pas - comme Max Jacob - et, de l'autre, il y avait à Paris des gens qui ne se cachaient pas, ne souffraient pas, qui exposaient, dont les œuvres étaient lues en public- comme Cocteau, Guitry et beaucoup d'autres » (FRANCK Dan, Monsieur Max, op. cit., dossier de presse non paginé). ${ }^{28}$ " Max Jacob est un "homme-frontière" : il explore constamment les "zones interdites" dans la société de son époque. Bien sûr, son destin est terrible : c'est un peu la rançon de ce parcours-là » (LECONTE Daniel, ibid.).

${ }^{29}$ SALMON André, « Note liminaire » dans JACOB Max, Cornet à dés II, Paris : Gallimard, 1955, p. 10.

${ }^{30}$ COCTEAU Jean, « Histoire du pantalon » dans Journal 1942-1945, op. cit., p. 567-569.

${ }^{31}$ Ibid. p. 566-567.

${ }^{32}$ Pour les controverses concernant le rôle de Picasso dans l'affaire Jacob, voir : ANDREU Pierre, Max Jacob, op. cit., p. 80 et Vie et Mort de Max Jacob, op. cit., le dernier chapitre ; Max Jacob et Picasso, catalogue sous la direction d'Hélène Seckel, Paris : Réunion des Musées Nationaux, 1994, note 25 , p. 277.

${ }^{33}$ COCTEAU Jean, « Si j'avais à me défendre, voici quels seraient les termes de ma défense », ms autographe, sd, dans Catalogue de vente Thierry Bodin, Paris : Thierry Bodin, mai 2007, p. 13. ${ }^{34}$ PAULHAN Jean, Choix de lettres, Paris : Gallimard, T. II, p. 351. Nous remercions Bernard Baillaud, président de la Société des lecteurs de Jean Paulhan de nous avoir permis de dater cette lettre circa fin mars 1944.

${ }^{35}$ DURAND Georges, « Max Jacob, texte d'une conférence prononcée le 21 mars 1944 au groupement paroissial des hommes de Saint-Benoît » dans Max Jacob, le poète pénitent de Saint-Benoîtsur-Loire, Max Jacob 1921-1944, Gien : Les documents du Val-d'Or (chez M. le curé), 5 mars 1945 , p. 9-18.

${ }^{36}$ Ibid., p. 17.

${ }^{37}$ SELLIER Philippe, « Chroniques de Port-Royal » dans Port-Royal et la littérature, Paris : Honoré Champion, 1999, p. 29-48.

${ }^{38}$ LEIRIS Michel, dans JACOB Max, In Memoriam Max Jacob, 1944, C'était il y a trente ans..., Mayenne : Les Amis de Max Jacob, 1974, p. 47 (article sans titre).

${ }^{39}$ CASSOU Jean, « Max Jacob et la parole », ibid., p. 21. 


\section{La mort de Max Jacob : réalité et représentations}

${ }^{40}$ DELÉTANG-TARDIF Yanette, « La mort d'un poète », Poésie 44, n 20, juillet-octobre 1944, repris dans MAX JACOB, le poète pénitent de Saint-Benoît-sur-Loire, Max Jacob 1921-1944, op. cit., p. 14-16.

${ }^{41}$ CLUZEL Raphaël, Le chemin de croix de Max Jacob, Paris : Richard Masse, 1983.

${ }^{42}$ LAGARDE Pierre, Max Jacob, mystique et martyr, Paris : Baudinière, 1944.

${ }^{43}$ Ibid. p. 9-10.

${ }^{44}$ CADOU René-Guy, « Images de Max Jacob (texte retrouvé) », dans JACOB Max, In Memoriam Max Jacob, 1944, C'était il y a trente ans..., op. cit., p. 20.

${ }^{45}$ SALMON André, Max Jacob, Poète, Peintre, Mystique et Homme de qualité, Paris : René Girard, 1927.

${ }^{46}$ Voir le dossier « Max Jacob personnage de roman » dans Les Cahiers Max Jacob, Pau : Presses Universitaires de Pau/ Les Amis de Max Jacob, nº 8, 2008, p. 11-77.

${ }^{47}$ FABUREAU Hubert, Max Jacob, Paris : La Nouvelle Revue Critique, 1935, p. 8-9.

${ }^{48}$ OXENHANDLER Neal, Looking for Heroes in postwar France, Albert Camus, Max Jacob, Simone Weil, Hanover and London: University Press of New England, Dartmouth College, 1996.

${ }^{49}$ SUSTRAC Patricia, compte rendu de ROSENBAUM Olga, Max Jacob « the integrity of the writer » dans Six authors in captivity-Literary responses to the Occupation of France during World War II, Bern : Peter Collier et Peter Lang (coll. Modern French Identities), vol. 54, 2006, dans Les Cahiers Max Jacob, Pau : Presses Universitaires de Pau/Les Amis de Max Jacob, n 7, 2007, p. 120.

${ }^{50}$ JACOB Max, Lettres à Roger Toulouse, 1937-1944, op. cit., p. 63.

${ }^{51}$ FRY Varian, La Liste noire, Paris : Plon, 1999, p. 258-263. 